\title{
- Current Updates in Plant Biology to Medicine to Healthcare Awareness in Malaysia
}

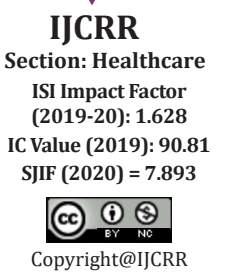

Section: Healthcare ISI Impact Factor (2019-20): 1.628 SJIF $(2020)=7.893$

Copyright@IJCRR

\section{Ajay G. Pise ${ }^{1}$, Ranjit Ambad²}

\author{
'Associate Professor, Dadasaheb Balpande College of Pharmacy, Besa, Nagpur, M.S., India. \\ Email: ajaygpise@gmail.com \\ ${ }^{2}$ Datta Meghe Medical College, Datta Meghe Institute of Medical Sciences, Sawangi Meghe, Wardha, M.S., India. \\ Email:ambad.sawan@gmail.com
}

Beyond the numbers, Malaysian universities have impacted local and international communities in various ways. Malaysia's research and development landscape has grown tremendously over the last decade. Moving forward, the focus is on research that brings positive impact to local and international communities also called as translational research. This special issue in the International Journal of Current Research and Review is happy to receive overwhelming response from Malaysia's researchers to publish their research via our journal platform. We are happy to release this special issue highlighting the research outcomes from Sarawak region of Malaysia. This special issue contains original research articles, reviews, and case studies from the field of basic science and medicine.

In the field of plant biology, the protein profiles of healthy and diseased $P$. nigrum roots was investigated and it is suggested that these can be potentially used as markers or be developed further to allow plants to gain systemic resistance against diseases and this, in turn, would help farmers to protect and improve the quality of the P. nigrum plants on their farm. Further, chemical profiling of honey from Heterotrigonaitamaon Borneo and evaluation for potential therapeutic properties. This study demonstrated that Borneo H. itamahoney is a potential source of antimicrobial and antioxidant agents. Another study focused on farmers' health has determined the occurrence of intestinal parasites in fresh retailed vegetables in Kuching and Kota Samarahan, Sarawak. This highlighted the potential of transmitting parasitic infections via vegetables especially if the vegetables are not prepared hygienically (e.g. washed, cooked, steamed, boiled) to render the parasites non-infectious for safe human consumption. It is suggested that high proportion of such infection risk may be reduced if farmers also take part in proper sanitation practices during vegetable production and transportation.

In the field of medicine, a case study outlined the importance of immediate accurate risk category stratification for early identification of those at risk in the primary care setting help minimize the risk of rabies infection and save a life. One more case study presented on the post-femoral fracture for its uniqueness and complexity of the concomitant complications.
Another group of researchers, provided a detailed review of existing knowledge on common simple red-eye cases. This will surely increase the reader confidence in managing the commonly observed red-eye cases and suggested that unresolved and complicated red-eye cases require ophthalmologist referral and further investigations in a timely manner. For eye health management, the study has compared the outcomes of transscleral diode cyclo-photocoagulation using short-duration versus long-duration burn treatment in Asian brown iris patients and found that both treatments reduced the intraocular pressure, and the number of antiglaucoma eye drops needed for glaucoma management.

In the survey study, the relationship between screening behaviour on colorectal cancer and health literacy among Sarawak's adult population was established and found that health literacy did not have any significant association with colorectal cancer screening behaviour of colorectal cancer. This may guide the development and formulation of successful health promotion and education to adopt healthy behaviour by focusing on health belief component apart from health literacy, thus reducing the cancer disparities and ensuring health equity. In the Malaysian adolescents, lifetime trauma, post-traumatic stress disorder symptoms, and early adolescent risk factors were evaluated, leading to poor physical health outcome. This study explains the roles of coping and psychological defence skills in traumatized adolescents exhibiting post-traumatic stress disorder symptoms and suggested that improving coping skills might directly improve traumatized adolescents' daily functioning.

Malaysian researchers are also focusing on the influence of recent COVID-19 pandemics. One study is included in this special issue, it intended to examine the knowledge, attitude, and practice towards COVID-19 and its preventive measures among Malaysians amid the conditional movement control order during the COVID-19 pandemic in Malaysia. We believe that this special issue has diverse scope and ranging from plant biology, medicine and healthcare awareness. This also highlights the progress of research in Malaysia and we are happy to disseminate it globally. 